\title{
Hierarchical, multimodal tomographic X-ray imaging at a superbend
}

\author{
M. Stampanoni*a,b, F. Marone ${ }^{\mathrm{a}}$, G. Mikuljan, ${ }^{\mathrm{a}}$ \\ K. Jefimovs ${ }^{\mathrm{c}}, \mathrm{P}$. Trtik ${ }^{\mathrm{c}}$, \\ J. Vila-Comamala ${ }^{\text {a }}$, C. David ${ }^{\text {a }}$, R. Abela ${ }^{\mathrm{a}}$ \\ ${ }^{\text {a }}$ Paul Scherrer Institut, 5232 Villigen, Switzerland; \\ ${ }^{\mathrm{b}}$ Institute for Biomedical Engineering, University and ETH Zürich, Zürich, Switzerland; \\ ${ }^{\mathrm{c}}$ Swiss Federal Laboratories for Material Testing and Research, 8600 Dübendorf, Switzerland;
}

\begin{abstract}
Over the last decade, synchrotron-based X-ray tomographic microscopy has established itself as a fundamental tool for non-invasive, quantitative investigations of a broad variety of samples, with application ranging from space research and materials science to biology and medicine. Thanks to the brilliance of modern third generation sources, voxel sizes in the micrometer range are routinely achieved by the major X-ray microtomography devices around the world, while the isotropic $100 \mathrm{~nm}$ barrier is reached and trespassed only by few instruments. The beamline for TOmographic Microscopy and Coherent rAdiology experimenTs (TOMCAT) of the Swiss Light Source at the Paul Scherrer Institut, operates a multimodal endstation which offers tomographic capabilities in the micrometer range in absorption contrast - of course as well as phase contrast imaging. Recently, the beamline has been equipped with a full field, hard X-ray microscope with a theoretical pixel size down to $30 \mathrm{~nm}$ and a field of view of 50 microns. The nanoscope performs well at X-ray energies between 8 and $12 \mathrm{keV}$, selected from the white beam of a $2.9 \mathrm{~T}$ superbend by a $[\mathrm{Ru} / \mathrm{C}]_{100}$ fixed exit multilayer monochromator. In this work we illustrate the experimental setup dedicated to the nanoscope, in particular the ad-hoc designed X-ray optics needed to produce a homogeneous, square illumination of the sample imaging plane as well as the magnifying zone plate. Tomographic reconstructions at $60 \mathrm{~nm}$ voxel size will be shown and discussed.
\end{abstract}

Keywords: hierarchical imaging, full field microscope, nanotomography, x-ray imaging, synchrotron CT

\section{INTRODUCTION}

The combination of the unique properties of X-ray microscopy (high-resolution, large penetration depth and chemical speciation) with advanced tomographic methods as well as the exceptional properties of third-generation synchrotron radiation sources allow to obtain volumetric information of a specimen with voxel size in the micrometer range. This is nowadays routinely achieved by the major synchrotron-based X-ray microtomography devices around the world [1-4].

Even rookie synchrotron-CT users, require today flexible endstations, capable to cover field of views (FOV) varying up to three orders of magnitude with corresponding spatial resolution and/or acquisition mode. Frequently, such users also demand an easy and fast swap between antipodal configurations, for instance between a $10 \mathrm{~mm}$ FOV with 10 microns resolution in phase contrast and a 50 microns FOV with $100 \mathrm{~nm}$ resolution in pure absorption, see Fig. 1.

The beamline for TOmographic Microscopy and Coherent rAdiology experimenTs (TOMCAT) of the Swiss Light Source provides this flexibility within a user friendly environment which allows even the less experienced user to be able to perform challenging experiments after a very short learning phase.

(*) email: marco.stampanoni@psi.ch, Tel.: +41 5631047 24, Fax.: +41 563104551.

Web: http://sls.web.psi.ch/view.php/beamlines/tomcat/index.html

Developments in X-Ray Tomography VI, edited by Stuart R. Stock,

Proc. of SPIE Vol. 7078, 70780V, $(2008) \cdot 0277-786 \times / 08 / \$ 18 \cdot$ doi: $10.1117 / 12.793759$

Proc. of SPIE Vol. 7078 70780V-1 


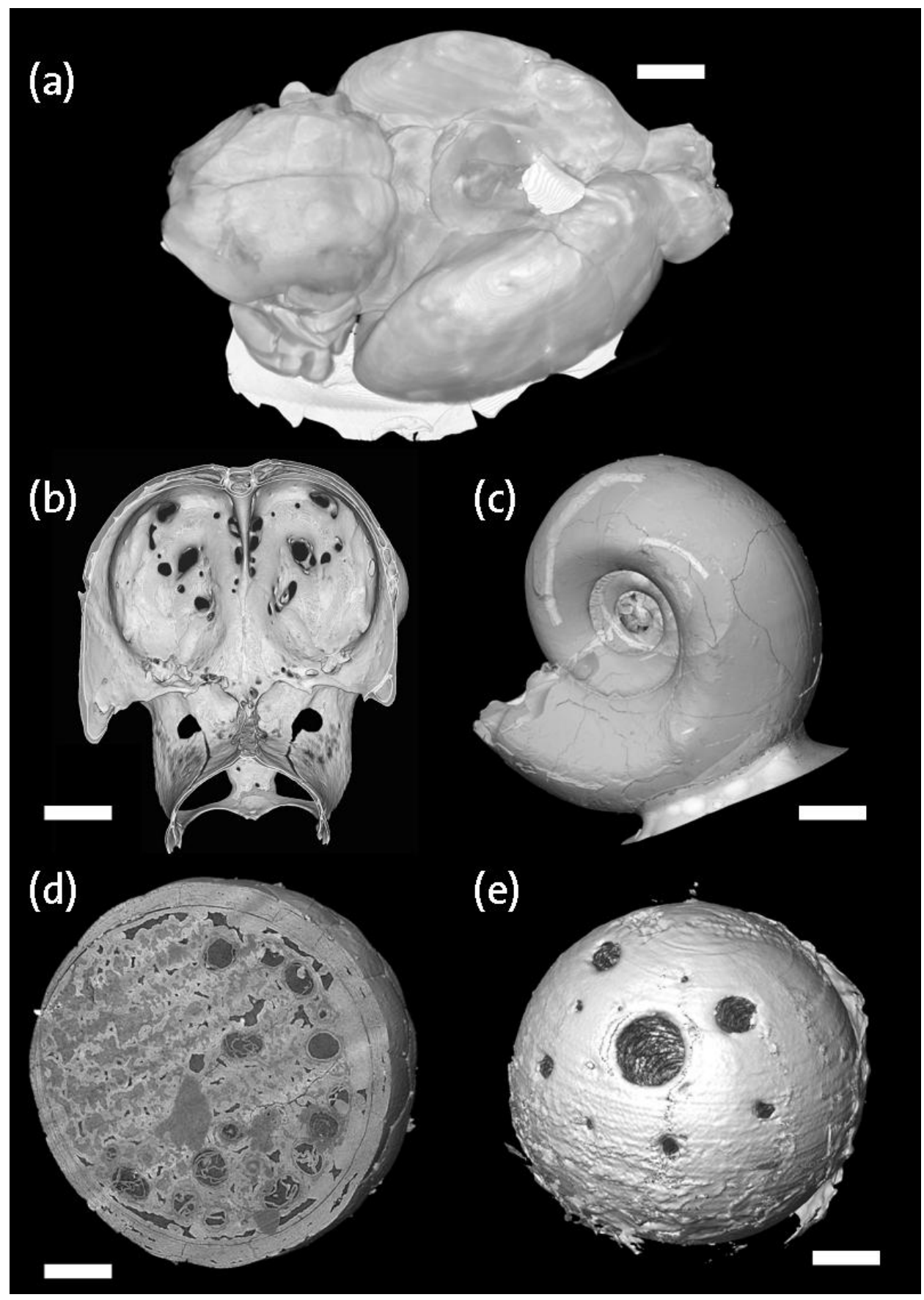

Fig. 1. Hierarchical, multimodal X-ray Tomographic Microscopy at TOMCAT. (a) Volume-rendered, differential phase contrast reconstruction of a whole mouse brain [9]. Field of view: $12 \mathrm{~mm}$, Pixel size 11.2 microns. Scale bar: $2 \mathrm{~mm}$. (b) Surface rendering of the olfactory plate of a bat (Pipistrellus pipistrellus) acquired with local tomography (sample courtesy Robert D. Martin, Field Museum, Chicago). Field of view $7.57 \mathrm{~mm}$, Pixel size 3.7 microns. Scale bar: $1 \mathrm{~mm}$. (c) Transparent surface rendering of a fossil ammonite (Elatmites elatme, from the Callovian (Middle Jurassic) age, 160 Million years old) acquired in edge-enhanced mode (sample courtesy of Larisa Doguzhaeva, Palaeontological Institute of the Russian Academy, Moscow). Field of view $3.78 \mathrm{~mm}$, Pixel size 1.85 microns. Scale bar: $0.5 \mathrm{~mm}$. (d) Orthosliced volume rendering of a fossil embryo (570 million years old), illustrating intact, deflated, and collapsed spheroidal and ovoidal interior structures, coated by several generations of cement [21]. Field of view $0.75 \mathrm{~mm}$, Pixel size 0.37 microns. Scale bar: $0.1 \mathrm{~mm}$. (e) Isosurface rendering of a $\mathrm{BaTiO}_{3}$ sphere of 22 microns diameter with calibration holes of 5, 2, 1, 0.5 and 0.25 microns diameter. Field of view: 30 microns, Pixel size $72 \mathrm{~nm}$. Scale bar: 5 microns. 
TOMCAT operates a multimodal endstation which offers tomographic capabilities in the micrometer range in absorption contrast as well as in phase contrast imaging. Absorption tomography can be performed between 8 and $45 \mathrm{keV}$ thanks to a fixed-exit double crystal multilayer monochromator.

Good transverse coherence length $\left(1_{\text {transV }} \times 1_{\text {transH }}=156 \mu \mathrm{m} \times 44 \mu \mathrm{m}\right.$ for $\lambda=1 \AA$ and a source-to-sample distance of 25 $\mathrm{m})$ assures excellent edge-enhancement all along the energy range. An in-house developed phase contrast algorithm (essentially based on the Transport of Intensity method) quickly allows to obtain phase contrast tomographic reconstruction of low absorbing, mostly homogenous objects at resolution around one micron [5, 6]. Full phase information is obtained via grating interferometry with FOV up to $12 \mathrm{~mm}$ and spatial resolution down to approximately 4 microns [7]. Detailed information on the technical specification and capabilities of the beamline can be found in this proceedings, see [8].

Here, as mentioned above, we will focus on the latest stage of the TOMCAT hierarchical multimodal imaging infrastructure, namely a full field X-ray microscope which reaches a 3D spatial resolution of less than 0.1 microns.

Reaching and trespassing the isotropic barrier of $100 \mathrm{~nm}$ remains a challenge and only few instruments mostly in the soft $\mathrm{X}$-ray range, i.e. for energies well below $10 \mathrm{keV}$ have this capability [10]. The most common approach to achieve sub $100 \mathrm{~nm}(2 \mathrm{D})$ resolution is full-field X-ray microscopy. The key optical elements are a condenser to provide illumination of the sample and an objective lens to produce a magnified image of the sample on the detector. The illumination should be as homogeneous and intense as possible and the numerical aperture should be matched to that of the objective lens in order to obtain optimum resolution [11]. Fresnel zone plates (FZPs) [12], tapered capillaries [13], mirrors [14] and combinations of these devices [15] are commonly used as condensers. However, these optics focus the beam into a spot, which is smaller than the field of view of the microscope. As a result they cannot provide a uniform but rather a Gaussian shaped illumination. This problem can be overcome by moving the condenser transverse to the direction of the beam during the exposure [15]. The use of beam-shapers with more complex optical functionality in the X-ray regime was first demonstrated by Di Fabrizio and co-workers [16]. However, the beam-shaper design is usually difficult since many unknown parameters of the beam have to be defined in advance and there are some restrictions owing to the fabrication limitations. A simple approach of a beam-shaper design was proposed and tested with a soft X-ray full-field microscope [17]. The idea of the design is to divide a conventional FZP into sectors, keeping the local spatial frequency within each sector constant. As a result, each sector will produce a flat-top illumination in the focal plane. The full-field microscope installed at the TOMCAT beamline uses an optimized design of such a beamshaper based on square gratings structured for hard X-rays. The shape of the produced illumination is square and can be designed to match the field of view of the microscope. In addition to the fulfillment of the basic X-ray microscopy requirements (numerical aperture matching and flat-top illumination) the beamshaper used here is extremely simple to install and align, enabling the endstation of the beamline to be turned from a "conventional" synchrotron-based microtomography device into a nanoscope with tomographic capabilities within a couple of hours. Furthermore, being each zone of the beamshaper equally contributing to the illumination of the image plane, this device is extremely insensitive to beam fluctuation and misalignments. Tomographic capabilites have been added to the full-field microscope by integrating the X-ray optics in the pre-existing microtomography endstation of the beamline.

\section{BEAMLINE PARAMETERS - OPTICAL COMPONENTS}

\subsection{Beamline parameters}

The TOMCAT beamline receives photons from a $2.9 \mathrm{~T}$ bending magnet, also dubbed superbend. Compared with the $1.4 \mathrm{~T}$ of the normal SLS bending magnets this device shifts the critical energy of the source from $5.4 \mathrm{keV}$ up to $11.1 \mathrm{keV}$ resulting in a considerable increase of flux at hard X-rays.

Monochromatic beam is extracted by a fixed-exit double crystal multilayer monochromator (DCMM) which covers an energy range between 8 and $45 \mathrm{keV}$. The DCMM is located in the front-end, at approximately $7 \mathrm{~m}$ from the source: this allows accepting a large angular divergence while keeping the optical element very compact. $[\mathrm{Ru} / \mathrm{C}]_{100}$ and $[\mathrm{W} / \mathrm{Si}]_{100}$ multilayer stripes have been coated $8 \mathrm{~mm}$ apart from each other on a Si(111) substrate (active area of $150 \times 50 \mathrm{~mm}^{2}$ ). As a result the energy bandwidth of the DCMM is a few percent when multilayer are used or $10^{-4}$ when the silicon is used. The slope errors figures in the direction along and across the optic are better than $0.5 \mu \mathrm{rad}$ RMS and $5 \mu \mathrm{rad}$ RMS respectively. Surface roughness is less than $0.3 \mathrm{~nm}$ RMS. When operating with multilayers the Bragg angle varies 
between $\theta_{B}=1.82^{\circ}$ and $\theta_{B}=0.265^{\circ}$ for energies ranging from $5 \mathrm{keV}$ up to $45 \mathrm{keV}$. For the low-energy settings, the power density is $80 \mathrm{~mW} / \mathrm{mm}^{2}$ and is dissipated via water side-cooling. The crystal optics are mounted on two independent high-precision goniometers. The first crystal has motorized pitch, roll, and horizontal translation (for stripes selection); the second crystal has the same degrees of freedom and, in addition, yaw and vertical translation. The whole system is positioned on a base plate that can be vertically adjusted [18].

For characterizing the nanoscope we selected $\mathrm{X}$-rays energies between 8 and $10 \mathrm{keV}$. For these values, the $[\mathrm{Ru} / \mathrm{C}]_{100}$ multilayer shows an energy spread of $2 \%$, which slightly affected the diffracting properties of the beamshaper as well as the imaging performance of the objective lens, as mentioned in [19].

\subsection{Beamshaper}

The design of the beam-shaping elements is based on a standard FZP. The original FZP is divided into square subfields, as shown in Fig. 2 and explained in details in [19].
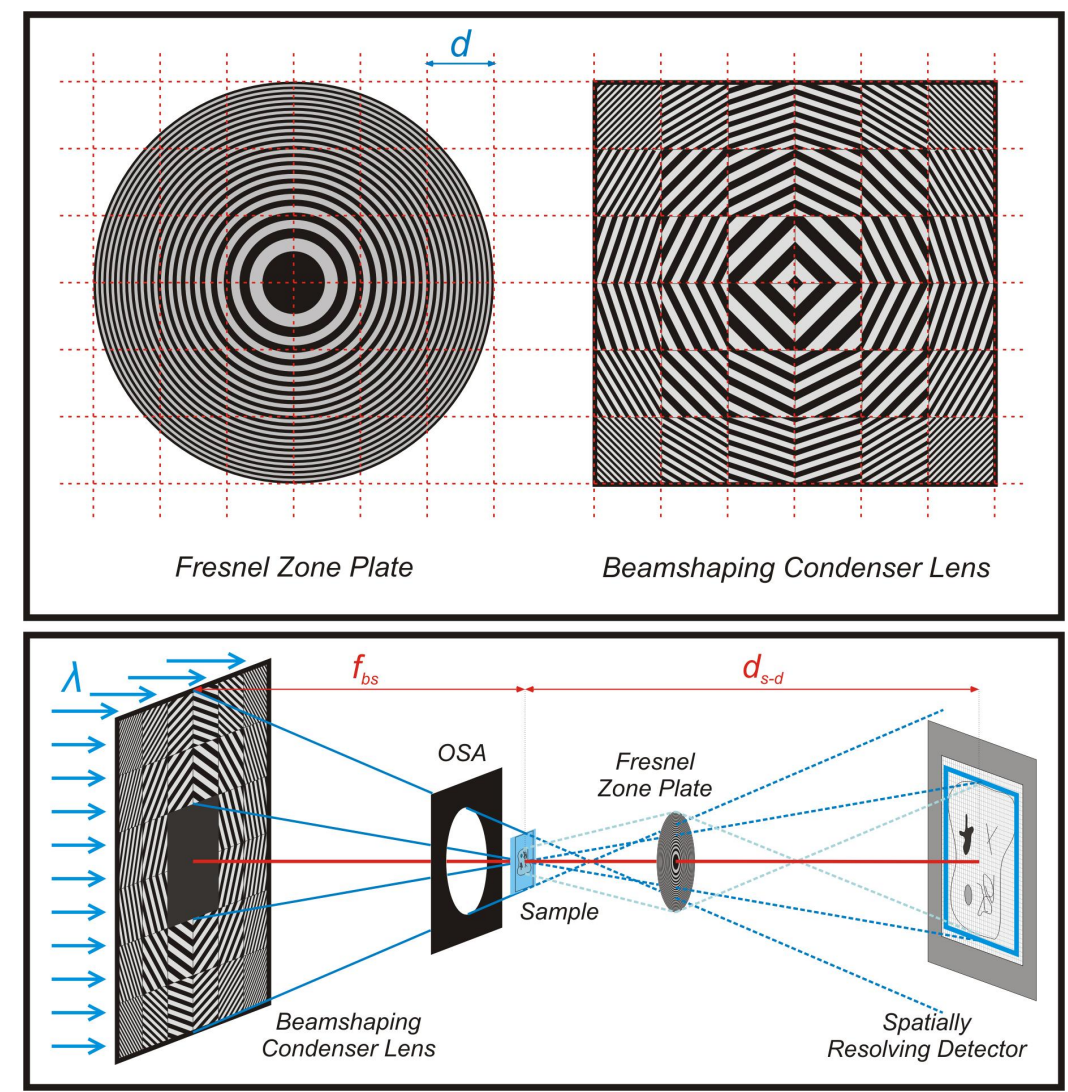

Fig. 2. Upper panel. Design of the beamshaper: the original FZP is divided into square subfields, each of them consisting of a linear grating with constant line orientation and period. Bottom panel: size, focal length and outermost zone width can be designed according to the experimental requirements. The geometry of the system can be efficiently adapted to the detector dimensions.

Each subfield consists of a linear grating with constant line orientation and period, which correspond to the local orientation and period of the structures of the FZP. As a result, the beam-shaper has the same focal length as the FZP, and the first diffraction order of every subgrating will form exactly coinciding square illumination in its focal plane with the illuminated area equal to the size of the gratings. The advantage over the design shown in [17] is that one can avoid tails of the illumination, which increases the intensity in the uniformly illuminated area. Another advantage of using the square shape of illumination is that usually the active area of the detector is also square. In this case the illumination of the beam-shaper can be perfectly matched to the detector size. 
Beam-shapers of different dimensions, from $0.5 \mathrm{~mm} \times 0.5 \mathrm{~mm}$ to $1.9 \mathrm{~mm} \times 1.9 \mathrm{~mm}$, with subfields of $50 \mu \mathrm{m} \times 50 \mu \mathrm{m}$ and with the outermost grating periods down to $100 \mathrm{~nm}(50 \mathrm{~nm}$ lines and spaces) have been fabricated. Despite using the same design, different materials and nanofabrication techniques are required for producing beam-shapers for soft and hard X-ray applications in order to achieve better efficiency. For hard X-rays the chosen material was gold, and 550-700 $\mathrm{nm}$-thick structures were prepared by electroplating into a polyimide mould as described in [20]. The main parameters of the gold beamshaper are summarized in Table 1.

In order to block the zero order through the condenser an opaque square central stop must be used. For hard X-rays the central stop was introduced in the system as an independent element. Several parameters such as the size of the element, size of the subgratings, period in the outermost gratings and size of the central stop are easy to address during the design in order to fulfill geometrical conditions of the specific experimental station (beam size, focal distance, sample size, active area of the detector). For instance, the size of the shadow formed by the central stop can be matched by the size of the beam-shaper illumination magnified by the objective lens.

Table 1. Main parameters of the gold beamshaper installed in the TOMCAT nanoscope for an energy of $8 \mathrm{keV}$.

\begin{tabular}{|ll|}
\hline Substrate & $\mathrm{Si} \sim 2 \mathrm{microns}$ \\
Substrate Transmission & $>97 \%$ \\
Structure height & from 0.7 to 1 micron \\
"Diameter" & $1700 \mu \mathrm{m}$ \\
Outer zone width & $70 \mathrm{~nm}$ \\
Spot size & $50 \mu \mathrm{m} \times 50 \mu \mathrm{m}$ \\
Theoretical Efficiency & $20 \%$ each zone \\
Gain Factor & 200 \\
Focal length & $1360 \mathrm{~mm}$ \\
Depth of focus & $200 \mu \mathrm{m}$ \\
Central stop size & $\sim 800 \mu \mathrm{m} \times 800 \mu \mathrm{m}$ \\
\hline
\end{tabular}

\subsection{Objective lens and detector}

As an objective lens a gold FZP with a $100 \mathrm{~nm}$ outermost zone and a diameter of 100 microns was used. The lens is mounted on a sand-filled stainless steel support which minimizes vibrations and shifts of the FZP, a critical issue in each "nano" experiment.

The X-ray detector was a Photonic Science Hystar Camera. This system is based on a scientific grade 2048x2048 TH7899 Thomson Chip with $14 \times 14 \mu \mathrm{m}^{2}$ pixel size. It has a full well capacity larger than 250 '000 electron/pixel. Readout at $200 \mathrm{kHz}$ with a noise of less than 10 electrons as well as a dark current of less than 0.5 electrons/pixel/second allow for a true 14 bit dynamic range via a 16 bit ADC. The chip sized is matched via 1:3 magnifying fiber optic taper (FOP). A $1.5 \mathrm{mg} / \mathrm{cm}^{2}$ Gadolinium Oxysulphide scintillator is deposited on the FOP and the final assembly results in an active input window of $9.5 \mathrm{~mm} \times 9.5 \mathrm{~mm}$ with an optical resolution of 4.7 microns.

\subsection{Experimental setup}

The nanoscope installed at the TOMCAT beamline is approximately 12 meters long. The beamshaper, acting as a beam condenser, is placed just after the exit window of the beamline, at 17 meters from the source, see Fig. 3a. The X-ray beam at that position is tailored to a size of $2 \times 2 \mathrm{~mm}^{2}$ by the exit slits of the beamline. Beamshaper and beamstopper are pre-mounted and aligned on a Linos frame and can be therefore easily inserted into the beampath. An xy-stage guarantees the fine adjustment. A thin vacuum pipe reduces beam losses due to air scattering. A JJ-slits pair is used as an adjustable order sorting aperture (OSA), see Fig. 3b. For our experiment the "pinhole" (which is actually a square) was set down to a size of $200 \mu \mathrm{m} \times 200 \mu \mathrm{m}$. The sample is mounted on an air-bearing rotation stage with and xy-centering device. The zone plate is fixed on a steel support and can be adjusted along three directions. Immediately downstream the zone plate, the high-resolution microscope (normally used at TOMCAT for routine experiments) can be inserted into 
the beampath. This allows the alignment of beamshaper, beamstopper and zone plate very quickly. The focal length of the FZP used for this experiment is $64.5 \mathrm{~mm}$ and $80.6 \mathrm{~mm}$ at $8 \mathrm{keV}$ and $10 \mathrm{keV}$ respectively. By positioning the 2D detector at distances larger than $10 \mathrm{~m}$ we can obtain X-ray magnifications up to $180 \mathrm{x}$. The detector is mounted on a second optical table, which can be remotely adjusted horizontally and vertically and manually displaced along the beam direction. An evacuated pipe connects both optical tables, see Fig. 3c.

We have been monitoring the stability of the system by acquiring X-ray radiographs of a high resolution mesh (Plano G2786A) over a period of 10 hours. Fig. 3d shows the vertical and horizontal shifts observed during a period of 2 hours. Shifts have been calculated by an in-house developed cross-correlation routine. The values remain within a range of \pm $100 \mathrm{~nm}$ within the first hour, which is the time usually needed for a tomographic scan. These values also correspond to the theoretical limit of the system, since the outermost zone period of the FZP is $100 \mathrm{~nm}$.

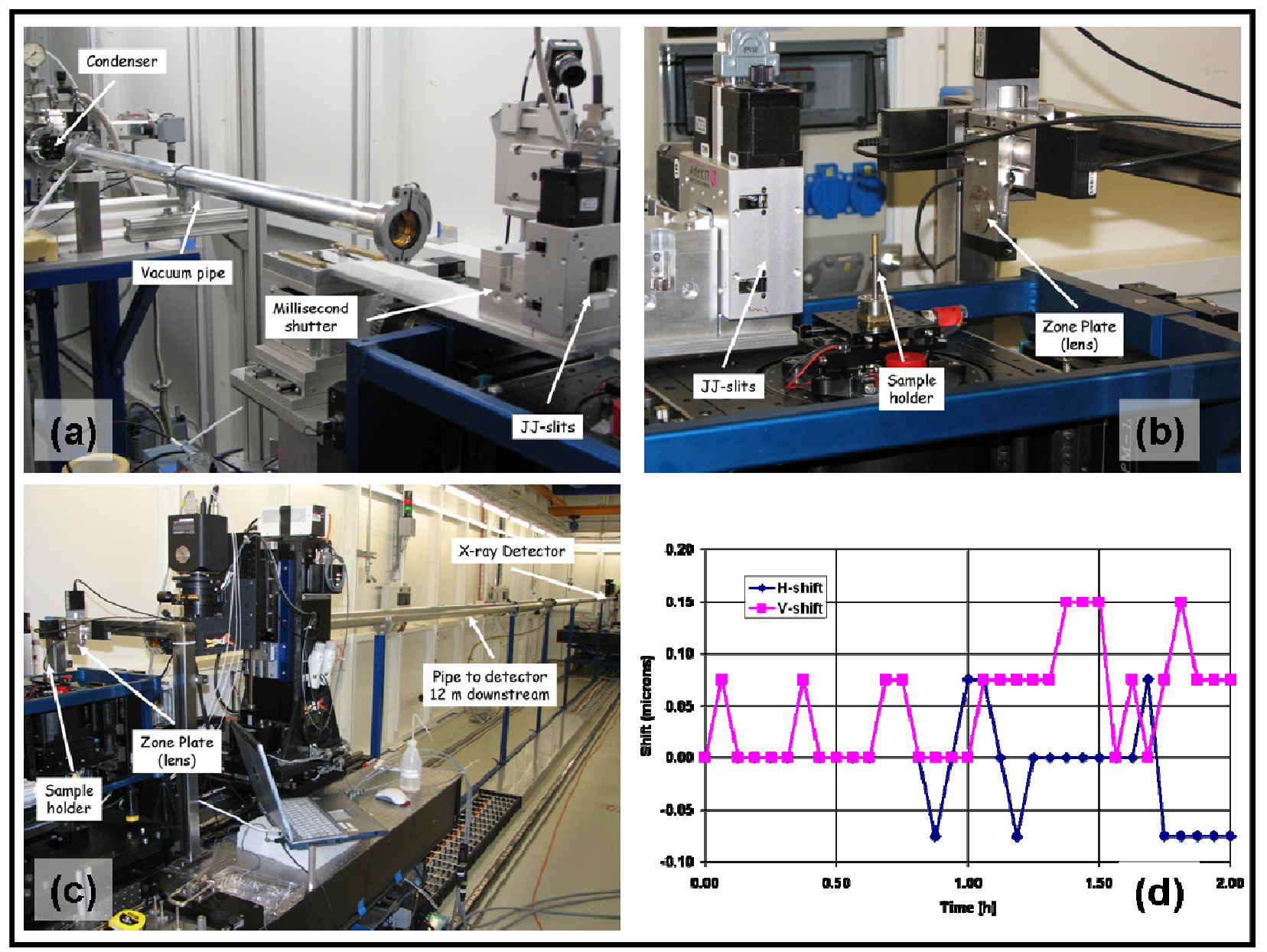

Fig. 3. Experimental setup of the nanoscope installed at the TOMCAT beamline. (a) The beamshaper is placed just after the exit window of the beamline. (b) A JJ-slits pair is used as a variable order sorting aperture. The zone plate is fixed on a steel support and can be adjusted along three directions. (c) The detector is positioned 10-12 meters downstream on a second optical table, which can be adjusted horizontally and vertically. (d) Plots of the vertical and horizontal shifts of the specimen observed at the sample position during a period of 2 hours. 


\section{SYSTEM PERFORMANCE}

\subsection{Radiographic projections.}

The radiographic imaging properties of the TOMCAT nanoscope have been characterized at $8 \mathrm{keV}$. For this purpose, as a test sample, we used a 20 microns diameter gold zoneplate with an outermost zone width of $100 \mathrm{~nm}$. Fig. 4 shows a resolution measurement for an X-ray projection. Fig. $4 \mathrm{~b}$ depicts the corresponding X-ray radiographic image (absorption image) of the test sample shown in Fig. 4a. A line profile over the central region of the zoneplate (Fig. 4c) shows that intensity oscillations at the frequency of $5 \mathrm{lp} /$ micron could be detected with sufficient contrast (Fig. 4d). The target resolution for this experiment was relatively relaxed, namely $100 \mathrm{~nm}$. The detector has been used in $2 \mathrm{x}$ binning mode, giving a theoretical pixel size of approximately $60 \mathrm{~nm}$. We were therefore sufficiently oversampling the detector plane.

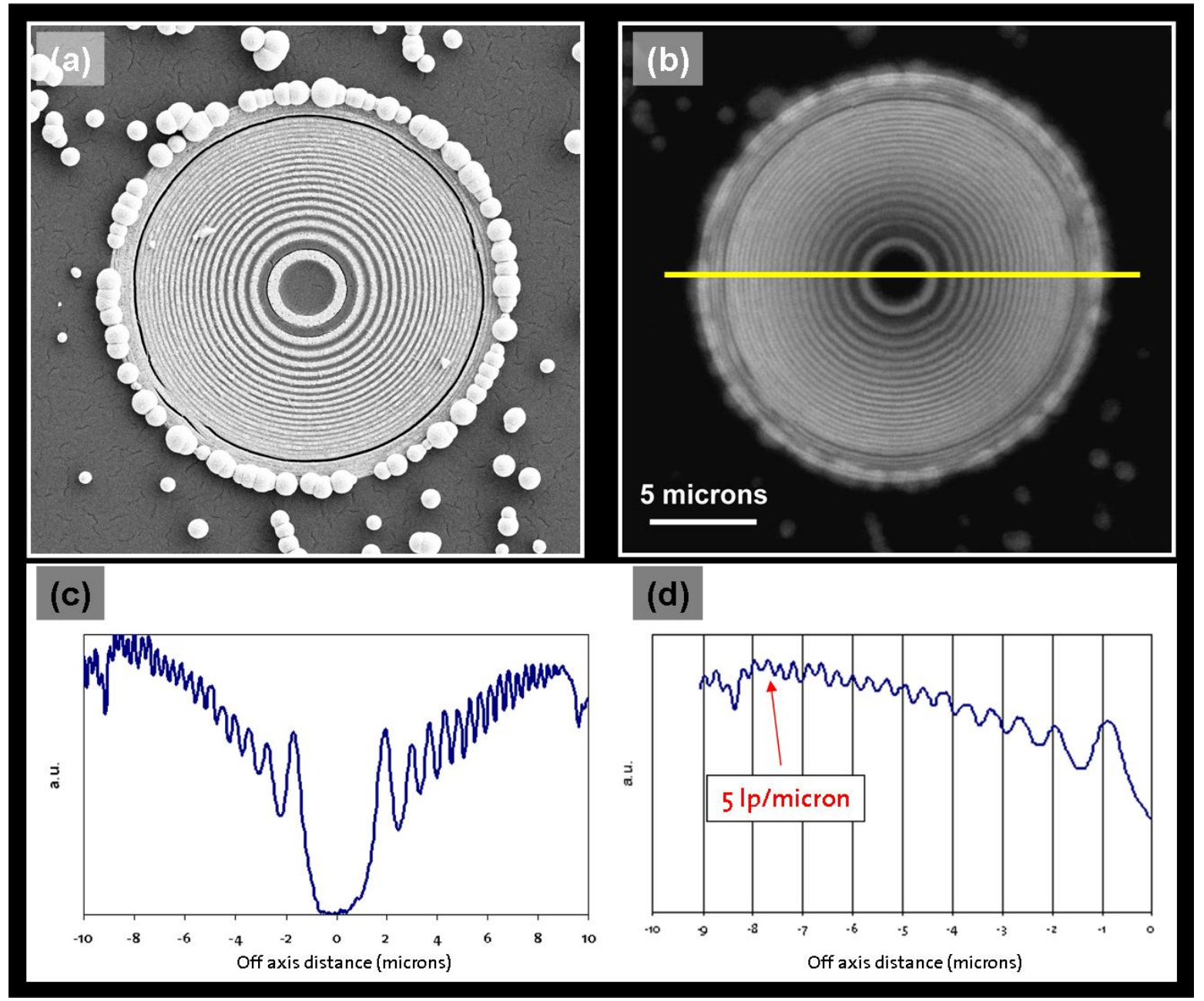

Fig. 4. (a) SEM of the reference sample: 20 microns diameter gold FZP with $100 \mathrm{~nm}$ outermost zone width. (b) Corresponding X-ray projection acquired at $8 \mathrm{keV}$. (c) Line profile and (d) zoom in into the profile, showing contrast at the target resolution $(100 \mathrm{~nm})$.

\subsection{Tomographic capabilities.}

The 3D imaging characteristics of the TOMCAT nanoscope have been characterized at $10 \mathrm{keV}$. At this energy the flux emerging from the bending magnet is larger and the absorption due to air in non-evacuated segments of the beamline is reduced. Therefore, the exposure time for single projections as well as stability issues could be minimized. 
We developed a test sample which is easy to align and ideally suited for tomographic investigations. It consists of a $\mathrm{BaTiO}_{3}$ sphere of 22 microns diameter with calibration holes of 5, 2, 1, 0.5 and 0.25 microns diameter, milled with focus ion beam at the FIB facility of EMPA, the Swiss Federal Laboratories for Material Testing and Research in Dübendorf, Switzerland, see Fig. 5a. The sphere has been coated by a thin gold layer to avoid charging during FIB milling. In the corresponding radiographic projection, see Fig. $5 \mathrm{~b}$, the gold coating is visible on the right side, while in the center the typical conical shape of the FIB holes are recognizable.

An isosurface rendering shows the entrance point of the reference apertures, see Fig. 5c. Holes with diameters of 5, 2, 1 and 0.5 microns on the surface of the sphere are clearly visible. The position of the 0.25 microns hole can be grasped as well. A virtual cut through the isosurface gives a more realistic view of the shape of the FIB holes. Due to redeposition of the removed material onto sidewalls of the holes they could not be milled with aspect ration larger than 5:1. Therefore the 5 microns hole was milled all the way through the sphere, while the 2 microns only reached the center of the sphere.

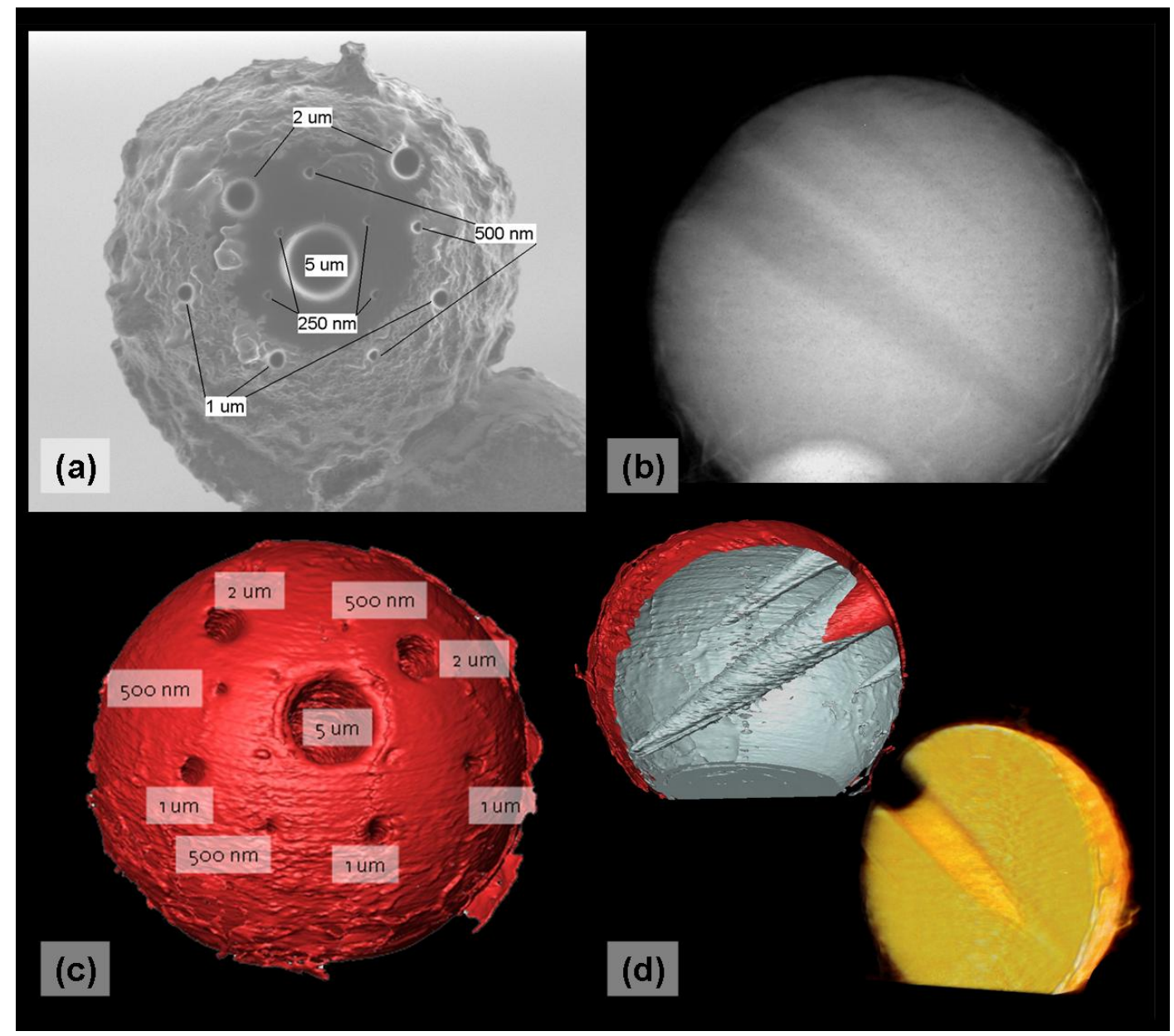

Fig. 5. (a) SEM of the reference sample used for nanotomography: a $\mathrm{BaTiO}_{3}$ sphere of 22 microns diameter with calibration holes of 5, 2, 1, 0.5 and 0.25 microns. (b) Corresponding X-ray projection acquired at $10 \mathrm{keV}$. (c) Surface rendering showing the entrance points of the reference holes. (d) Virtual cuts through the test sample, showing the typical cone shape of the FIB-milled holes. 


\section{DISCUSSION AND OUTLOOK}

The present work illustrates the latest instrumental development at the TOMCAT beamline, aiming at improving the spatial resolution down to $100 \mathrm{~nm}$ or slightly below. The nanoscope design was intended to be as simple to use as possible in order to be easily integrated into the imaging framework of the beamline, which offers field of views up to several $\mathrm{mm}$ in both absorption and phase contrast mode

We have shown that with a novel, ad-hoc designed beamshaper based on diffractive optics, it is possible to obtain topflat illumination of large image areas, allowing to "switch on" the nanoscope module at the beamline very quickly.

The first preliminary results are matching the design goals: we have been aiming for resolution down to $100 \mathrm{~nm}$ with sufficient oversampling and Fig. 4 shows that this target has been achieved. The stability of the system, usually one of the most critical issues in this kind of experiments, is sufficient for moving from simple full field microscopy to tomographic applications. However, we have been reaching the mechanical limits of the present system, which has been actually designed originally for micro- and not for nano-tomography. Tomographic data sets will need additional software corrections (mainly achieved via 2D cross correlation and image registration) in order to be optimally reconstructed.

In the next future we will further improve the stability and fine tune our registration algorithms. A new, larger beamshaper will collect a beam area of up to $3 \times 3 \mathrm{~mm}^{2}$ and tailor it down to $50 \times 50$ microns ${ }^{2}$, therefore augmenting the flux at the sample position by a factor of 4 . We also plan to update the present detector with a more efficient system which should result in a reduction of the exposure time by at least a factor of 2 .

Finally, our efforts will be focused in the tentative of producing phase contrast images with the nanoscope. A preliminary experiment shows that we can detects edge-enhanced signals as shown in Fig. 6.

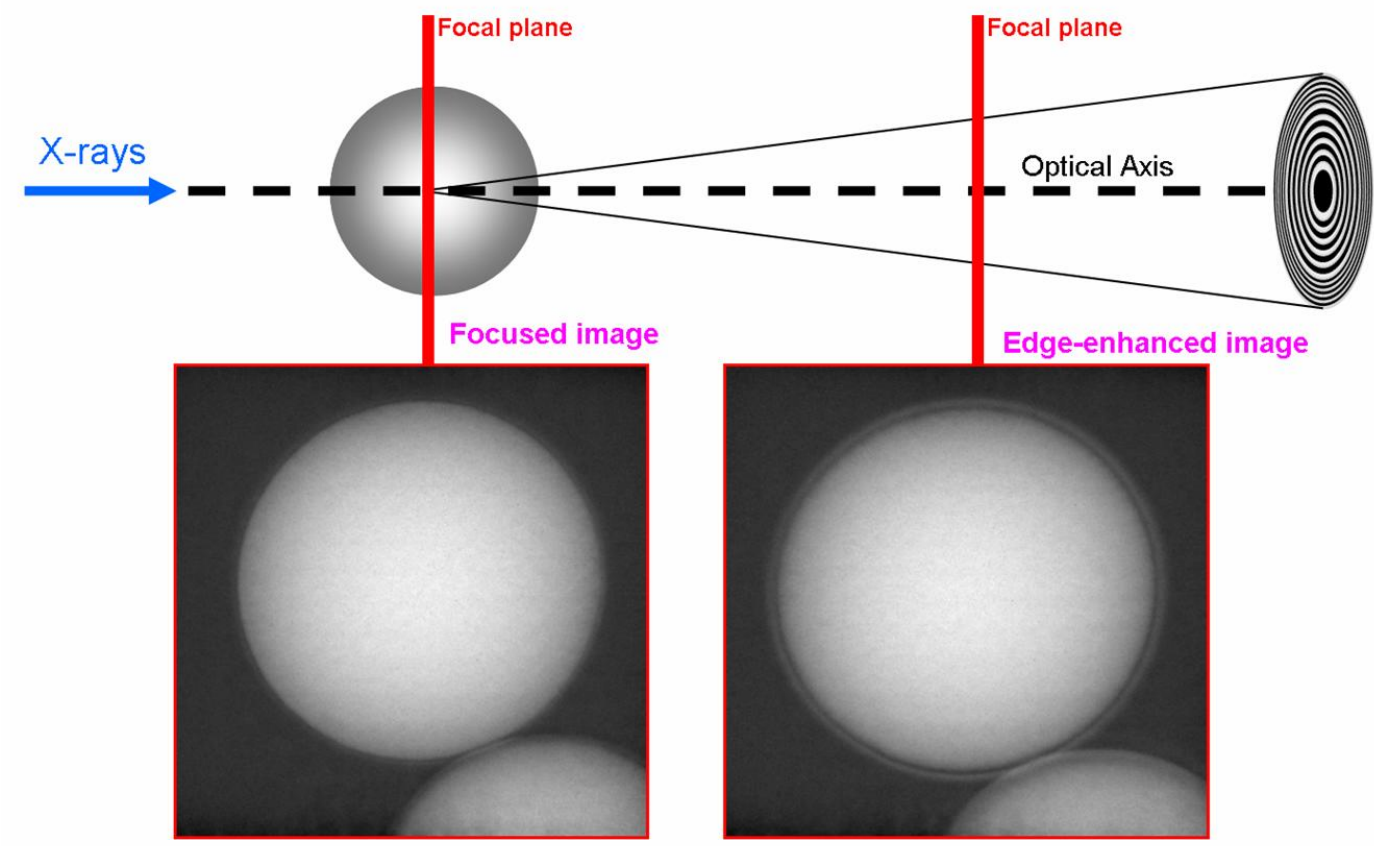

Fig. 6: X-ray nanoradiography of a $\mathrm{BaTiO}_{3}$ sphere of 22 microns diameter acquired in the focal plane of the zone plate (left) and downstream, in the so called edge-enhanced regime (right). The first Fresnel fringe appears as a ring since the resolution of the system is sufficiently high to oversample the interfringes space.

This means that our system preserves the coherence and we can therefore potentially use either Transport of Intensity [22,23] based methods or Grating Interferometry [7] for detecting phase signals. 


\section{REFERENCES}

[1] Lee R., Lai B., Yun W., Mancini D., Cai Z., "X-ray microtomography as a fast three-dimensional imaging technology using a CCD camera coupled wiht a $\mathrm{CdWO}_{4}$ single-crystal scintillator", Proc. SPIE 3149, 257-264 (1997)

[2] Dowd B., Campell G. H., Marr R. B., Nagarkar V., Tipnis S., Axe L., Siddons D. P., "Developments in synchrotron X-ray computed microtomography at the National Synchrotron Light Source", Proc. SPIE 3772, 224-236 (1999)

[3] Weitkamp T., Raven C., Snigirev. A., "Imaging and microtomography facility at the ESRF beamline ID22", Proc. SPIE 3772, 311-317 (1999)

[4] Beckmann F, Vollbrandt J, Donath T, Schmitz HW, Schreyer A, "Neutron and synchrotron radiation tomography: New tools for materials science at the GKSS-Research Center", Nuclear Instruments and Methods in Physics Research Section A 542 (3), 279-282 (2005)

[5] Grošo A., Abela A. , Stampanoni M., "Implementation of a fast method for high resolution phase contrast tomography", Optics Express 14, 8103-8110 (2006)

[6] Friis E. M., Crane P. R., Pedersen K. R., Bengtson S. , Donoghue P. C. J. , Grimm G. W. , Stampanoni M., "Phasecontrast X-ray microtomography links Cretaceous seeds with Gnetales and Bennettitales”, Nature 450, 549-552 (2007)

[7] T. Weitkamp, A. Diaz, C. David, F. Pfeiffer, M. Stampanoni, P. Cloetens, E. Ziegler, "X-ray phase imaging with a grating interferometer", Optics Express 13, 6296-6304 (2005),

[8] Marone F., Hintermüller C., McDonald S., Abela R., Isenegger A., Stampanoni M., "X-ray Tomographic Microscopy at TOMCAT", Proc. SPIE 7078, (2008)

[9] McDonald S., Marone F. and Stampanoni M., In preparation

[10] Meyer-Ilse W, Hamamoto D, Nair A, Lelievre SA, Denbeaux G, Johnson L, Pearson AL, Yager D, Legros MA, Larabell, "High resolution protein localization using soft X-ray microscopy", Journal of Microscopy 201, 395-403, (2001)

[11] Born, M. \& Wolf, E., "Principles of Optics", 7th ed., Cambridge University Press, 595 (1999)

[12] Anderson E. H., Olynick D. L., Harteneck B., Veklwrov E., Denbenaux G., Chao W. L., Lucero A., Johnson L. \& Attwood D., "Nanofabrication and diffractive optics for high-resolution x-ray applications", J. Vac. Sci. Technol. B., 18, 2970-2975 (2000)

[13] Yin G. C., Song Y. F., Tang M. T., Chen F. R. Liang, K. S., Duewer F. W., Feser M., Yun, W. \& Shieh, H. P. D., "30 nm resolution $\mathrm{X}$-ray imaging at $8 \mathrm{keV}$ using third order diffraction of a zone plate lens objective in a transmission microscope", Appl. Phys. Lett. 89, 221122 (2006)

[14] Rau C., Crecea V., Peterson K. M., Jemian P. R., Richter C. P., Neuhäusler U., Schneider G., Yu X., Braun P. V. and Robinson I. K., IPAP Conference Series, Vol.7 (2005)

[15] Niemann B., Guttmann P., Rehbein S. and Knöchel C., „, Concept and realization of the novel rotating condensermonochromator at the Gottingen TXM at BESSY II “, J. Phys. IV, 104, 273-276 (2003)

[16] Di Fabrizio E., Cabrini S., Cojoc D., Romanato F., Businaro L., Altissimo M., Kaulich B.,Wilhein T., Susini J., De Vittorio M., Vitale E., Gigli G. and Cingolani R., "Shaping X-rays by diffractive coded nano-optics", Microelectron. Eng. 67-68, 87-95 (2003)

[17] Vogt U., Lindblom M., Charalambous P., Kaulich B. \& Wilhein T., „, Condenser for Koehler-like illumination in transmission X-ray microscopes at undulator sources “, Optics Letters 31, 1465-1467 (2006)

[18] Stampanoni M., Groso A., Isenegger A., Mikuljan G., Chen Q., Bertrand A., Henein S., Betemps R., Frommherz U., Böhler P., Meister D., Lange M. and Abela R., "Trends in synchrotron-based tomographic imaging: the SLS experience", Proc. SPIE 6318, 63180M (2006)

[19] Jefimovs K., Vila-Comamala J., Stampanoni M., Kaulich B., David C., "Beam-shaping condenser lenses for fullfield transmission X-ray microscopy”, Journal of Synchrotron Radiation 15, 106-108 (2008)

[20] Jefimovs K., Bunk O., Pfeiffer F., Grolimund D., van der Veen J. F. \& David C., „, Fabrication of Fresnel zone plates for hard X-rays“, Microelectron. Eng. 84, 1467-1470 (2007)

[21] Hagadorn J.W., Xiao S.H., Donoghue P C. J., Bengtson S., Gostling N. J., Pawlowska M., Raff E.C., Raff R. A., Turner F.R., Chongyu Y., Zhou C., Yuan X., McFeely MB., Stampanoni M., Nealson K.H, ,Cellular and subcellular structure of neoproterozoic animal embryos“, Science 314 (5797), 291-294 (2006) 
[22] Op de Beeck, M. D. V. Dyck, and W. Coene, "Wave function reconstruction in HRTEM: the parabola method", Ultramicroscopy 64, 167 (1996)

[23] Cloetens, P., Ludwig W., J. Baruchel, D. V. Dyck, J. V. Landuyt, J.-P. Guigay, and M. Schlenker, "Holotomography: Quantitative phase tomography with micrometer resolution using hard synchrotron radiation $\mathrm{x}$ rays", Appl. Phys. Lett. 75, 2912 (1999) 